\title{
A brief overview of novel approaches to FDG PET imaging and quantification
}

\author{
Søren Hess • Björn A. Blomberg • Rajan Rakheja • \\ Kent Friedman • Thomas C. Kwee • \\ Poul Flemming Høilund-Carlsen · Abass Alavi
}

Received: 28 February 2014/ Accepted: 25 April 2014/Published online: 3 June 2014

(C) Italian Association of Nuclear Medicine and Molecular Imaging 2014

\begin{abstract}
The widespread implementation of hybrid FDG $\mathrm{PET} / \mathrm{CT}$ worldwide has brought about a paradigm shift in the use of diagnostic imaging - molecular imaging combining morphological and functional data is now at the forefront of patient management in many clinical settings, not only for initial diagnosis, staging, monitoring of response to treatment and detection of recurrence, but also for prognostication and disease characterization. Although developments have focused particularly on the qualitative visual analysis of images, FDG PET allows elaborate quantification, which should be explored much more in the future and ideally standardized worldwide. In this brief overview, we outline the state of the art of novel and quantitative approaches to FDG imaging, i.e. standard uptake value, partial volume effect and partial volume correction, multiple-time-point imaging, assessment of global disease burden and PET/MRI, all of which we expect to see evolving as powerful tools in the coming
\end{abstract}

Color figures online at 10.1007/s40336-014-0062-2.

S. Hess $(\bowtie) \cdot$ B. A. Blomberg · P. F. Høilund-Carlsen

Department of Nuclear Medicine, Odense University Hospital, Soender Boulevard 29, 5000 Odense, Denmark

e-mail: soeren.hess@ rsyd.dk

B. A. Blomberg - T. C. Kwee

Department of Radiology and Nuclear Medicine,

University Medical Centre, Utrecht, The Netherlands

R. Rakheja · K. Friedman

Nuclear Medicine Section, Department of Radiology, New York University School of Medicine, New York, NY, USA

\section{A. Alavi}

Division of Nuclear Medicine, University of Pennsylvania Perelman School of Medicine, Hospital of the University of Pennsylvania, Philadelphia, PA, USA years and enhancing sensitivity and specificity in variety of clinical settings, in which FDG PET imaging has not yet shown its full potential.

Keywords Molecular imaging · Positron emission tomography $\cdot$ PET $\cdot$ PET/CT $\cdot$ Quantification

\section{Introduction}

In 2007, the Society of Nuclear Medicine and Molecular Imaging defined molecular imaging as "the visualization, characterization and measurement of biological processes at the molecular and cellular levels in humans and other living systems. Molecular imaging typically consists of two- or three-dimensional imaging as well as quantification over time. The techniques used include radiotracer imaging/nuclear medicine, MR imaging, MR spectroscopy, optical imaging, ultrasound and others" [1]. Today, molecular imaging is paving the way for personalized approaches to patient management, the mantra of modern medicine and positron emission tomography (PET) imaging with ${ }^{18}$ F-fluoro-deoxyglucose (FDG) is at the heart of this evolution. Although the technology behind FDG PET was actually developed in the 1970s, unprecedented developments in the past decade, not least the commercial availability of hybrid PET/computed tomography (PET/ $\mathrm{CT}$ ), have led to its more widespread clinical use in contemporary medicine. It currently finds clinical as well as research applications in several of the major domains of human health and disease, e.g. neurology, oncology, cardiology and infection/inflammation [2].

Neurology has played a key role in PET imaging from the inception of this technology; indeed, this role dates all the way back to the very first experiences with positron 


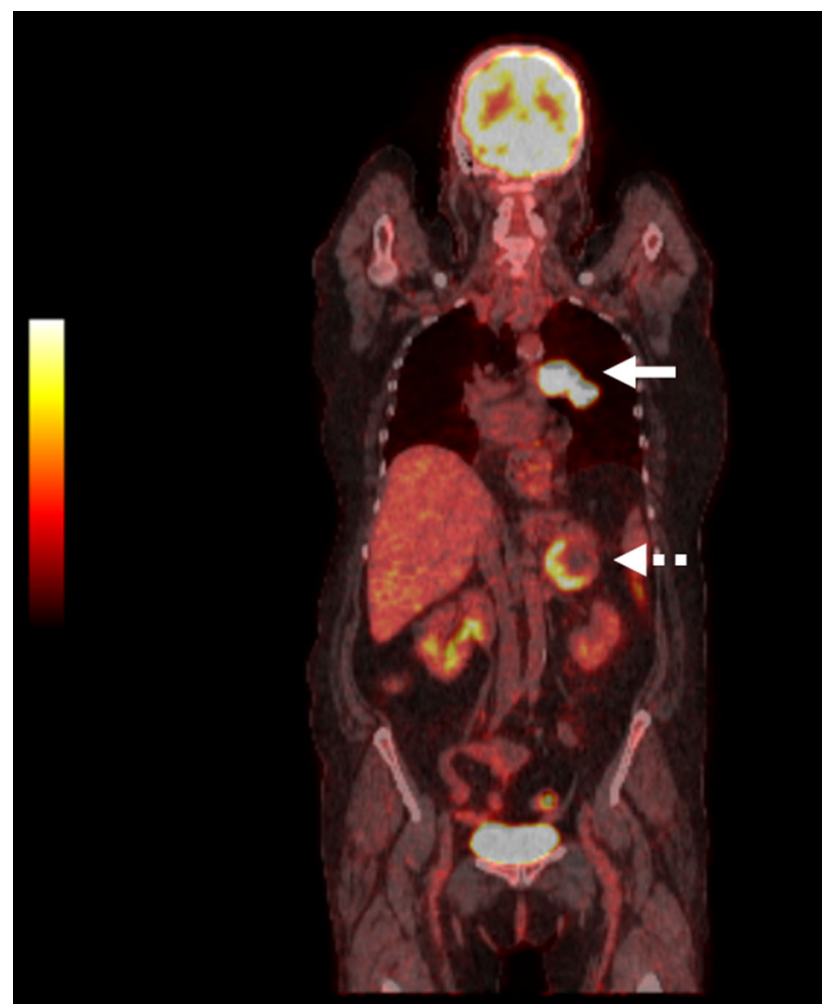

Fig. 1 60-Year-old female with suspected lung cancer referred for initial staging. FDG PET/CT shows FDG uptake in a perihilar tumour in the left lung (arrow) and the left adrenal gland (dotted arrow) (color figure online)

emission studies of brain tumours in the early 1950s. Later, from the 1970s onwards, PET was used extensively in the characterization of a multitude of neurological and psychiatric disorders; today, it is used particularly in epilepsy, Alzheimer's disease, Parkinson's disease and psychiatric disorders [2,3].

Applications in malignant diseases are diverse, and FDG PET is now utilized in many different cancers, a development driven by the concept of malignant hypermetabolism initially proposed by Warburg in the 1930s. The metabolic trapping of FDG in hypermetabolic cells due to up-regulation of GLUT transporters, increased levels of hexokinase and decreased levels of glucose-6-phosphatase, provides a sensitive approach to cancer diagnostics. FDG PET and FDG PET/CT are now increasingly employed in most malignant diseases, not only for diagnostic purposes, but also, increasingly, for pretherapeutic staging (Fig. 1), recurrence detection, response assessment and prognostication. PET has been shown to change patient management in a substantial proportion of cases, and there is increasing evidence of FDG providing valuable prognostic information [2,4]. Furthermore, there is a growing awareness of the importance of incidental findings, as well as an increasing interest in locating the primary malignancy in cancers of unknown origin (Fig. 2) and paraneoplastic syndromes [2].

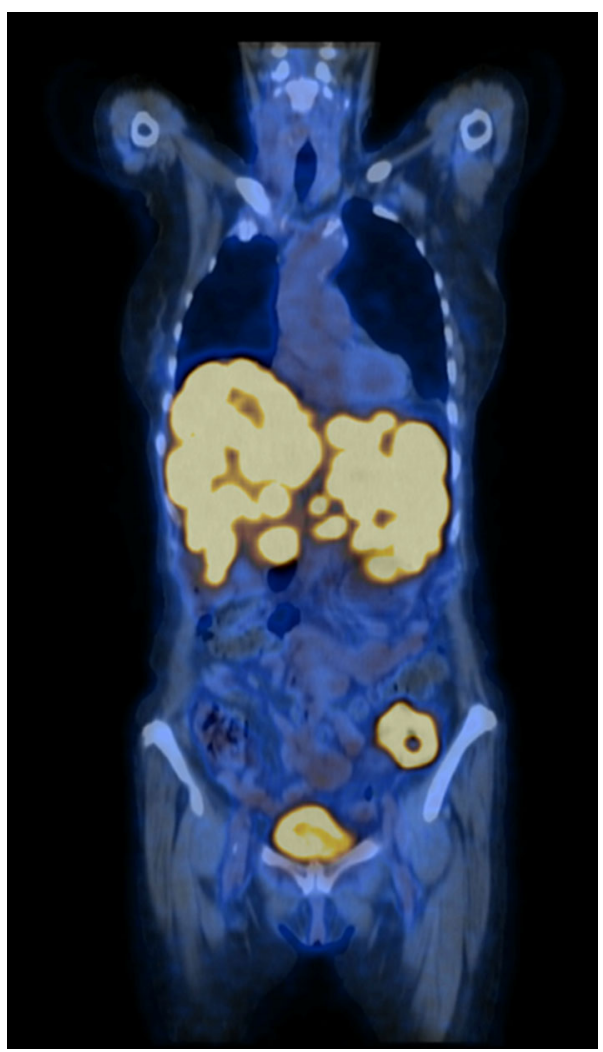

Fig. 2 Computed tomography imaging acquired after a traumatic injury revealed multiple liver metastases of unknown primary. Subsequent whole-body FDG PET/CT imaging located the primary tumour in the sigmoid colon (color figure online)

Assessment of myocardial viability and assessment of the inflammatory component of atherosclerosis and the vulnerable plaque are the main applications in cardiovascular disease, and this is in line with what are probably the most promising fields of application of FDG: infection and inflammation. In this context, the applications are versatile and novel indications emerge frequently. In infection, they include diagnosis of: fever and bacteremia of unknown origin, fever in the immunocompromised host, bone infections, infection of vascular grafts, etc., whereas inflammatory diseases in which FDG can play a role include, among others, vasculitis, sarcoidosis, inflammatory bowel disease (IBD), inflammatory joint disorders, low back pain, psoriasis, venous thromboembolism and musculoskeletal disorders (Fig. 3). The common denominator of these very different clinical entities is a newly found appreciation of systemic components not limited to the classical target organs, components that make the use of a sensitive whole-body survey desirable. This holds true, in particular, for the assessment of disease activity and the assessment of treatment response, which are difficult or impossible using conventional morphological modalities because the changes in morphology lag behind the molecular responses seen with FDG PET [2]. 
Fig. 3 19-Year-old female referred for FDG PET/CT due to fever of unknown origin. FDG PET/CT shows increased FDG uptake in the terminal ileum (dotted arrow) and in the pelvis (arrow). Surgery confirmed Crohn's disease with a fistula and an abscess (color figure online)
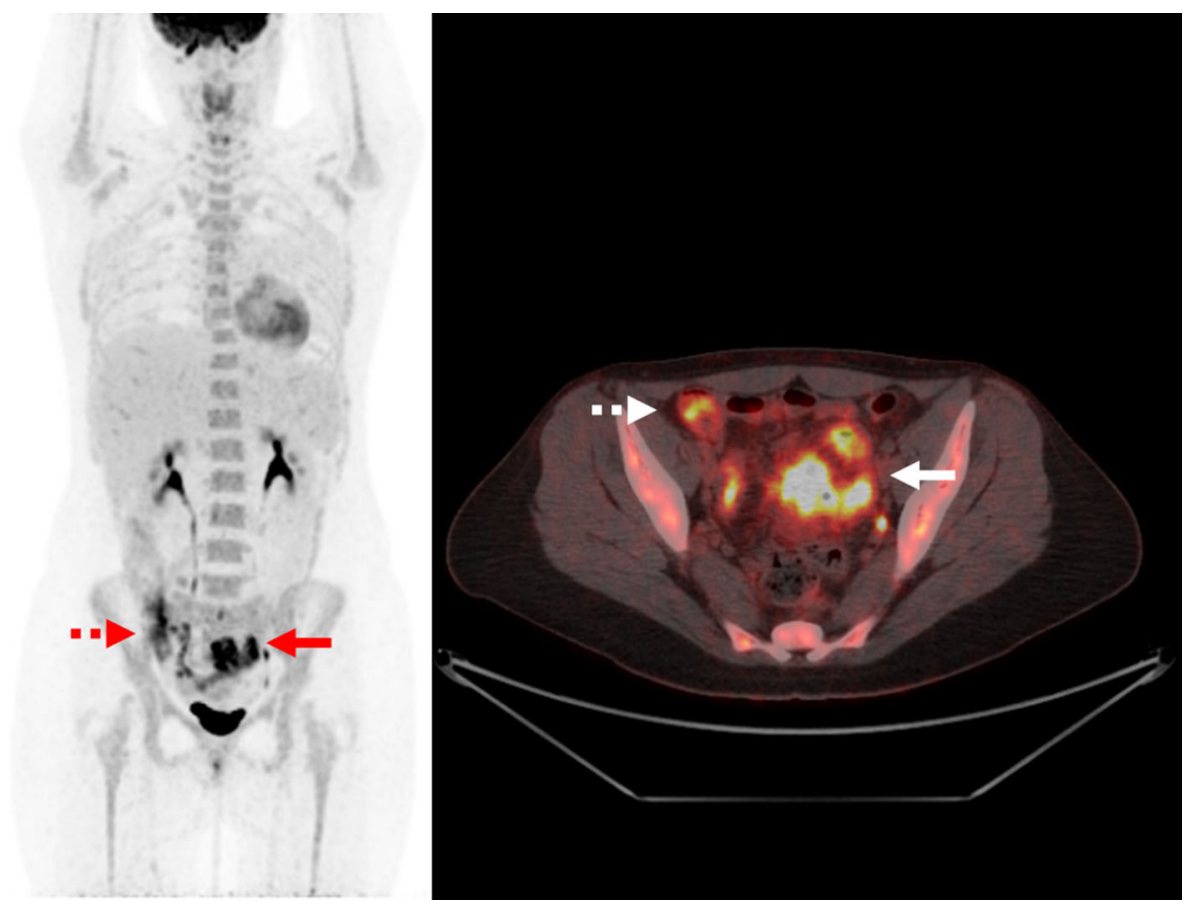

Even though developments and applications in this field have largely concerned qualitative visual analysis of images, the unique features of PET technology in general, and FDG PET in particular, open the way for elaborate quantitative methods which should be explored, utilized and emphasized much more in the future. Ideally, quantification methods should be standardized worldwide to facilitate multicentre trials, allow direct comparison between centres and lay the foundations for personalized therapy. Serious concern has been expressed over the present widespread application and uncritical use of suboptimal or inadequate quantification methods [5].

Therefore, it is increasingly being recognized that proper quantification of PET data is necessary in order to obtain much more objective and accurate assessment of disease extent and therapy response, as well as prognostic information, especially in systemic diseases. In this short overview, we present the state of the art of novel approaches to FDG imaging, focusing on the most important technical and quantitative developments, i.e. standard uptake value, partial volume effect (PVE) and partial volume correction (PVC), multiple-time-point imaging and assessment of global disease burden. Several papers thoroughly reviewing the promising potential of quantitative analysis in FDG PET/CT have emerged in recent years [610]. Some key points from these will be highlighted below and discussed in relation to applications in various disease areas. We conclude this overview with a brief look at emerging developments in FDG PET/magnetic resonance imaging (MRI), a modality that may offer some advantages over PET/CT, but also presents challenges.
Standard uptake value (SUV)

The rate of glucose metabolism can be measured using mathematical tracer kinetic models and may be considered a gold standard for the quantification of FDG avidity. However, it requires dynamic data acquisition and continuous arterial blood sampling, which is a complex and time-consuming procedure. Also, the field of view in most dynamic methods is limited to one bed position, which is adequate for limited areas of interest, such as brain studies, but challenging in the case of whole-body assessment of oncological lesions. Thus, simpler semi-quantitative methods have been developed and become increasingly widespread; the most widely employed of these is SUV measurement. The SUV reflects the degree of FDG uptake in a region or volume of interest (ROI or VOI), as measured at a certain time after FDG injection and normalized to the dose injected and the whole-body distribution, typically by taking into account the patient's body weight. These calculations are usually performed automatically by software indigenous to the scanner and, thus, readily available to most PET centres. The method is technically simple, requiring neither blood samples nor dynamic acquisition. Furthermore, a linear correlation has been demonstrated, with correlation coefficients of $0.84-0.91$ between SUV and glucose metabolism determined by kinetic modelling [11, 12]. Usually, maximum SUV (SUVmax, or the maximum singlevoxel SUV in a ROI) and mean SUV (SUVmean, the average SUV of all the pixels in a ROI) are reported.

Several injection- and patient-related factors and technical issues influence SUV measurements. Injection-related factors include extravasation at the injection site, residual 
activity in the syringe, and decay between the time of dose measurement and the time of injection. All these factors, if not properly corrected for, tend to lower the calculated SUV. The patient, on the other hand, is a variable source of error. For example, although the SUV is normalized to body weight, tissue composition is also a rather important consideration, since adipose tissue exhibits less metabolic activity and, consequently, less FDG uptake than other tissues. Thus, in obese patients with a higher proportion of body fat, the SUV may overestimate the metabolic activity occurring in non-adipose tissue. Several studies have shown superior results with SUVs instead normalized to body surface area or lean body mass. It has been argued that the differences between normalization methods are small, except in obese patients. Therefore, in view of the escalating trend of obesity, appropriate corrections will need to be developed in the future. In addition, obese patients are especially prone to elevated blood glucose levels, which also influence the SUV. Hyperinsulinaemia is a key feature in type-2 diabetes. This leads to increased glycolysis in muscle and adipose tissue, which in turn reduces the SUV in other tissues due to lower uptake of FDG. Thus, most PET centres postpone FDG imaging in patients with plasma glucose levels above $150-200 \mathrm{mg} / \mathrm{dL}$ $(\sim 8-11 \mathrm{mmol} / \mathrm{L})$. Interestingly, this seems to apply only to malignant tissue and not to inflammatory processes, as described by Zhuang et al. [13] who found no effect on SUV in benign lesions even with blood glucose levels up to $250 \mathrm{mg} / \mathrm{dL}(\sim 14 \mathrm{mmol} / \mathrm{L})$.

There is also a host of technical issues to be considered. Motion of organs or lesions may lead to misalignment of emission and anatomical data. As a result, gated acquisition or other measures must be applied to ensure valid quantitative data, especially in the assessment of lesions affected by respiratory movements. There is also a need for standardization of reconstruction methods and attenuation correction protocols since, for example, highly smoothed reconstruction may underestimate SUV. Since the tracer may be non-uniformly distributed within the ROI, as is the case with heterogeneous tumours, the SUVmax tends to overestimate the true mean value of a lesion. Measuring the SUVmean is much more operator dependent and timeconsuming, and so, automated algorithms facilitating calculation are in great demand. Finally, the SUV is usually measured only for a subset of lesions, potentially giving rise to sampling errors. A detailed description of the errors associated with SUV measurement and advice for acquisition are provided in European guidelines for tumour PET imaging [14].

Despite the potential pitfalls and drawbacks, the use of the SUV is widespread, especially for prognostication and response evaluation in various oncological settings as summarized in a recent review by Tomasi et al. [15]. They pointed out that a main problem lies in multicentre comparison and that the parameter, if computed in the same centre using the same settings, may be rather robust. The versatility of SUV is underlined by emerging clinical evidence for its use in response assessment and prognostication in various cancers, both common and rare, e.g. nonsmall cell lung cancer, breast cancer, gastro-oesophageal cancer, lymphoma, osteosarcomas and malignant mesothelioma [15-17]. Also, outside the field of oncology, SUV is being used for response evaluation and disease characterization. In a recent study, Nanni et al. [18] showed the feasibility and superiority of using changes in SUVmax, as compared to c-reactive protein, to establish and monitor response in patients with haematogenous infective spondylodiscitis. This is in line with our own experience (Fig. 4). In inflammation, a study by Groshar et al. [19] found SUVmax to be a reliable parameter for indicating the grade and severity of disease activity in IBD patients. Recently, Rondina et al. [20] showed decreasing SUVmax over time in patients with deep venous thrombosis, which suggests that there could be a role for FDG PET/CT in differentiating acute, active disease from chronic, inactive stages, with implications for therapeutic strategy [2].

Partial volume effect (PVE) and partial volume correction (PVC)

The term PVE refers to several phenomena, mostly related to scanner resolution, which cause the measured intensity of lesions to differ from their real values. First of all, the 3D blurring caused by the spatial resolution of the PET scanner causes smaller sources to appear larger in size but dimmer in signal intensity due to a spillover effect from the source to its surroundings. This blurring effect is even more pronounced with moving lesions, e.g. lesions that move due to respiration or cardiac motion. The best achievable resolution of contemporary whole-body PET scanners is $3-4 \mathrm{~mm}$ in a controlled laboratory setting using standardized phantoms, but in clinical practice the actual resolution of reconstructed images is considerably less due to limitations in the statistics of acquired data and in reconstruction algorithms. Lesion-to-background contrast decreases with decreasing lesion size and ultimately disappears completely. Thus, it is not possible to accurately measure SUV in lesions smaller than $\sim 2-3$ times the spatial resolution of the PET scanner, as defined by the full width at half maximum. Furthermore, PET images are sampled using a voxel grid, but the voxel rarely matches the actual contour of a given lesion or the distribution of tracer in general. Since the signal intensity in a voxel is a mean of the tissues included, most voxels include different types of tissue. Altogether, for lesions smaller than the reconstructed spatial resolution, which some studies suggest are 
Fig. 4 Spondylodiscitis in Th56 and L4-5 diagnosed and followed up with FDG PET/CT imaging. Fused sagittal (top left) and transaxial (bottom left) FDG $\mathrm{PET} / \mathrm{CT}$ at baseline and after 4 weeks of treatment showing marked improvement in FDG uptake (right column). SUVmax decreased significantly at both locations: from 9.9 to 3.8 in the thoracic spine and from 8.3 to 2.9 in the lumbar spine (color figure online)
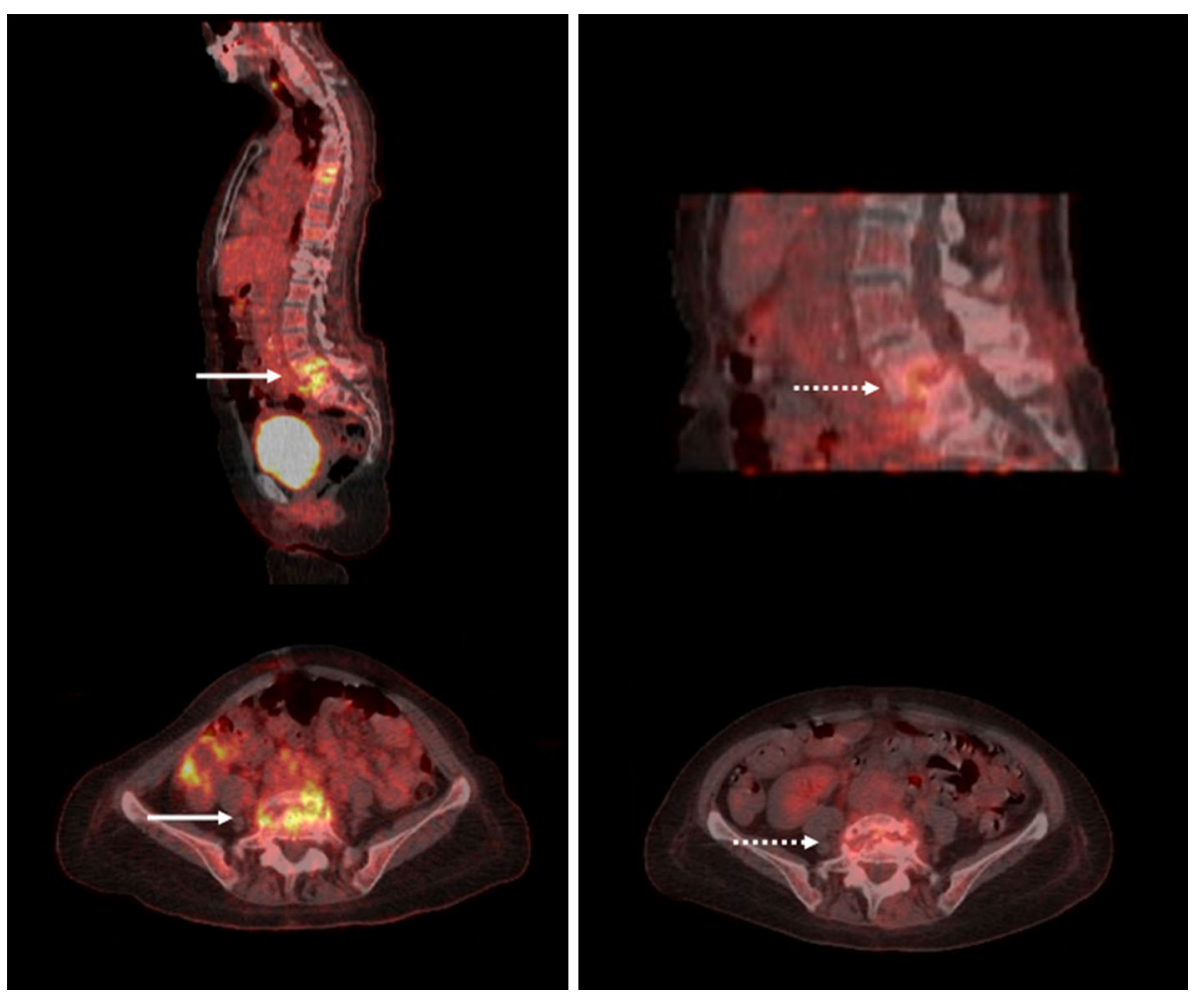

those less than $2 \mathrm{~cm}$ in size, PVE may result in a more than $50 \%$ underestimation of the true FDG concentration. Thus, $\mathrm{PVC}$ is of the utmost importance.

There are several methods that can be used to correct for PVE. Although we will not explore these methods in depth here, we nevertheless emphasize that the importance of PVC cannot be overstated. Results from lung cancer patients have shown PVC to increase the accuracy from 55 to $89 \%$ in lesions $<2 \mathrm{~cm}$ in size [21], while in breast cancer, correcting the SUV for PVE and blood glucose levels provided the highest diagnostic accuracy among various quantitative PET methods [22]. Similarly, a study comparing ${ }^{110 \mathrm{~m}}$ In-DTPA-D-Phe ${ }^{1}$-octreotide PET images with ${ }^{111}$ In-DTPA-D-Phe ${ }^{1}$-octreotide SPECT images found a great improvement with regard to the detection of small tumours and quantification when using PET with PVC [23].

\section{Multiple-time-point imaging}

FDG uptake and distribution is a dynamic mechanism rather than a static process. A study investigating the dynamic changes in FDG uptake and clearance in normal tissue in 30 patients who underwent FDG PET imaging at 1,2 and $3 \mathrm{~h}$ after injection of FDG demonstrated significantly decreased activity over time in some organs (the aortic blood pool, liver, spleen, lungs, pancreas, lymph nodes and skeletal muscle), while the activity increased in the myocardium and bones, and did not change at all in other organs (parotid gland, thyroid gland and prostate gland) [24]. This study showed that only a better understanding of the pharmacodynamics of FDG in healthy tissue and in pathological tissue will allow us to exploit the full capability of FDG PET imaging, not only with regard to visual assessment, but also quantification, since the above variations may substantially influence SUV measurements. Several studies assessing the dynamics of FDG uptake in various tissues have found that the plateau for FDG uptake in malignant cells is reached late, at approximately 3-4 h postinjection, while normal and inflammatory cells reach an equilibrium in the first hour after injection and gradually decrease thereafter [25, 26] (Fig. 5). This suggests that currently employed scan protocols may yield SUVmax values that are much lower than the true ones and therefore undermine the use of arbitrarily defined thresholds, e.g. an SUVmax of 2.5, for differentiating between benign and malignant findings.

The dynamics of FDG distribution may be utilized to increase both sensitivity and specificity, the latter being a significant clinical problem, especially in oncological imaging, where uptake in infectious processes and inflammatory tissues can generate false-positive results. As described in the first part of this review, FDG is taken up by glucose transporters, which are often up-regulated in hypermetabolic cells, regardless of whether they are benign or malignant. After phosphorylation by hexokinase, FDG6-phosphate is trapped intracellularly, as the fluorine atom prevents further metabolism in Krebs' cycle. Shortages of 
glucose-6-phosphatase (the enzyme responsible for the dephosphorylation that allows FDG to leave the cell) cause FDG-6-phosphate to accumulate in malignant tissues. By contrast, inflammation is characterized by many mononuclear cells expressing high levels of GLUT transporters, hexokinase and glucose-6-phosphatase. Thus, their uptake mimics that of malignant cells, but the clearance of FDG from the cell occurs much faster.

These characteristics make it possible to differentiate benign from malignant lesions by employing dual- or multiple-time-point imaging, since malignant cells keep

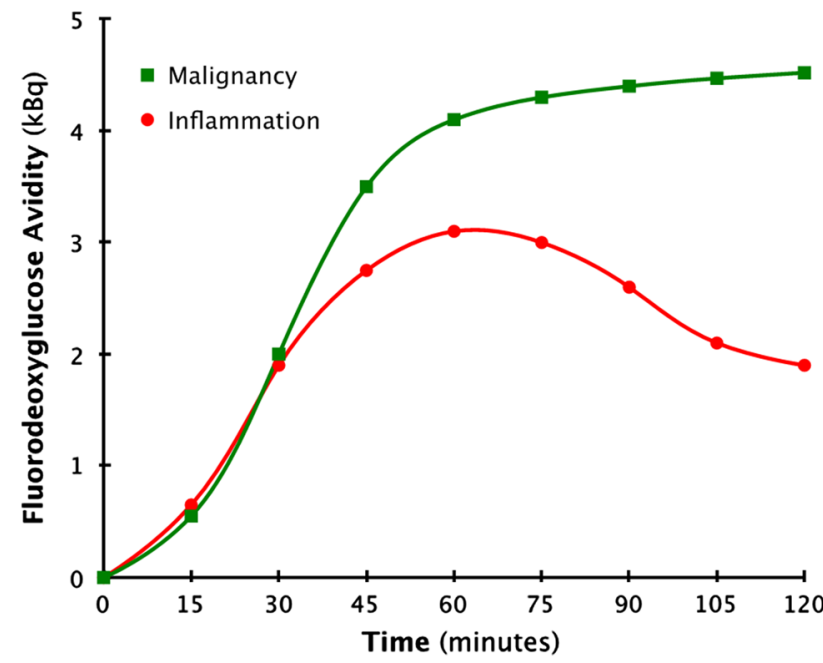

Fig. 5 Dynamic pattern of FDG avidity in inflammatory and malignant tissue (color figure online) accumulating FDG overtime while benign ones show a decrease in uptake in the same time frame. This trend was first hypothesized more than a decade ago and has since been confirmed by several studies, which showed it to increase both sensitivity due to higher target-to-background ratio and specificity by allowing better differentiation between benign and malignant tissue. This is especially noteworthy since new approaches to imaging techniques usually involve a trade-off between sensitivity and specificity. To date, multiple-time-point imaging has been successfully employed in the assessment of several malignant diseases, including lung [27] (Fig. 6), breast [28], head and neck [29] and colorectal cancer [30].

Global disease burden

Alavi et al., in the early 1980s, introduced the concept of global metabolic activity for brain assessment in patients with Alzheimer's disease and age-matched controls [31, 32]. They demonstrated significant differences between the two groups by multiplying segmented brain volumes (determined by MRI) by the measured mean cerebral metabolic rates for glucose and suggested that this method might be adapted to other settings. In general, the idea is to combine volumetric and metabolic data into a summary measure termed global disease burden, whole-body metabolic burden, or total lesion glycolysis, instead of evaluating these parameters independently and on a per-lesion basis. Semi-automated software has since become available, rendering both tissue segmentation and global disease
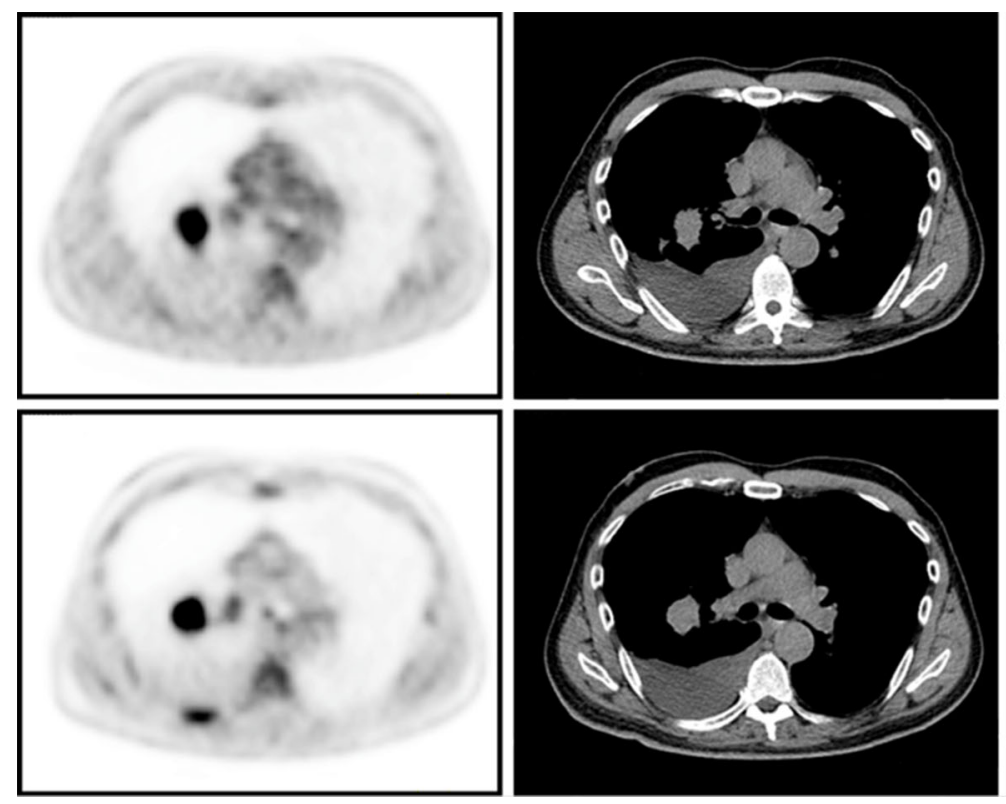
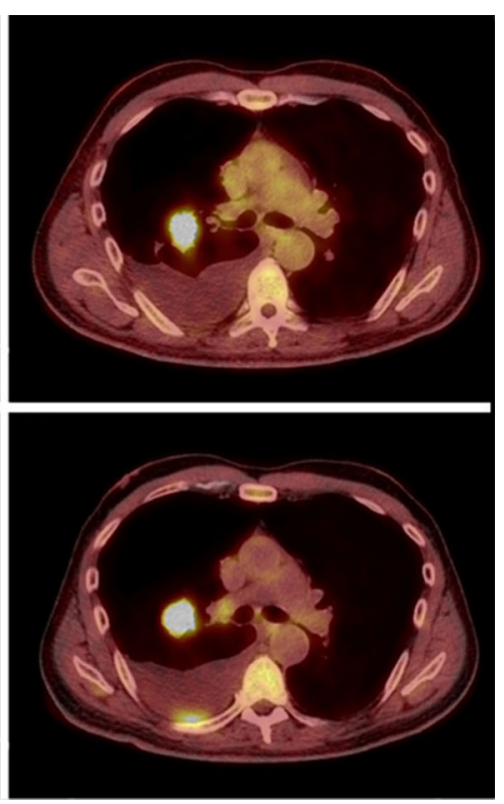

Fig. 6 Preoperative dual time-point FDG PET/CT imaging in a patient with suspected lung cancer. One hour after FDG administration, an FDG-avid mass in the right lung (top row) was visible.
Delayed imaging at 3 hours revealed an additional FDG-avid lesion in the right pleura, suggesting a pleural metastasis (bottom row) (color figure online) 


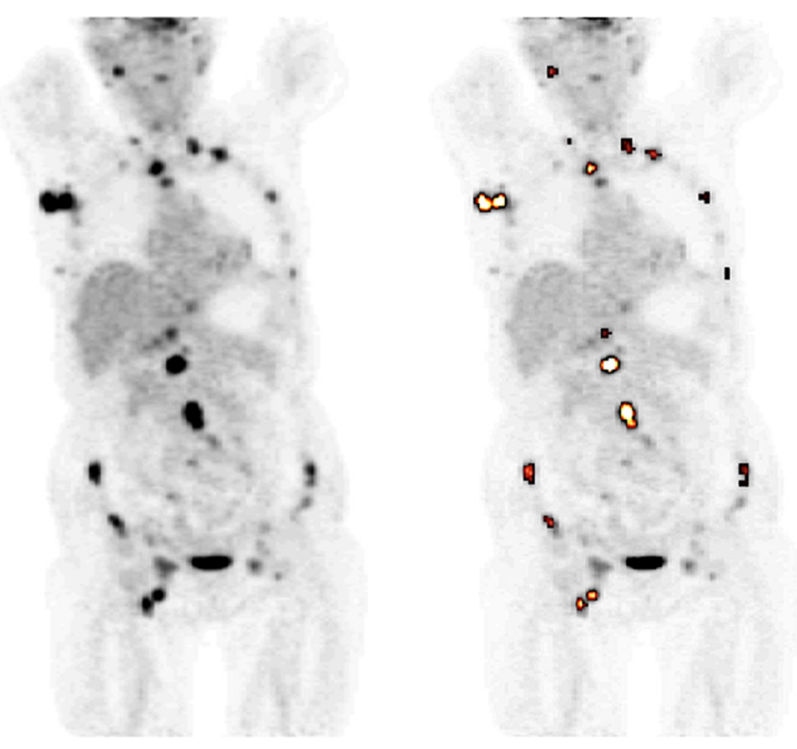

Fig. 7 FDG PET imaging detected multiple FDG-avid lesions in a patient with lymphoma disease $(l e f t)$. Semi-automated segmentation allowed calculation of the global disease burden (right) (color figure online)

assessment easier and faster than when manually performed. Preliminary results have shown good reproducibility and a high level of agreement with manual measurements, indicating reasonable accuracy.

Some authors have found one such method to be feasible in clinical practice in malignant mesothelioma [33] and in sarcoidosis [34]. Using commercial software (ROVER, ABX GmbH, Germany), FDG-avid lesions were segmented by adaptive thresholding, generating the metabolically active volume (MAV) and the partial volume corrected SUVmean (cSUVmean). Subsequently, the partial volume corrected metabolic volume product (cMVP) was calculated as cMVPmean $=$ MAV $\times$ cSUVmean. Finally, a global disease activity score was obtained as the sum of the cMVPs in all FDGavid lesions throughout the body. Semi-automated global disease assessment of this type may provide an efficient, reproducible and accurate means of quantifying widespread disease (Fig. 7), which could be especially valuable for evaluating treatment response, not only in many cancer types, but also in systemic inflammatory diseases, atherosclerosis, etc. Thus, a recent article [35] reviewing the current literature on metabolic tumour volume and total glycolysis in various solid tumours concluded that "both metabolic tumour volume and total lesion glycolysis have the potential to become valuable as prognostic biomarkers for survival outcome, clinical staging and response to both neoadjuvant and concurrent therapies".

\section{PET/MRI}

Ten years after the introduction of the first clinical PET/CT scanner, another multimodality imaging technology appeared: combined PET/MRI. The advantages of MRI over CT are its excellent soft tissue contrast, the lack of ionizing radiation and the possibility of providing additional functional tissue information using different MRI methods such as diffusion-weighted MRI, and perfusion MRI, which may aid in the evaluation of disease and in therapy planning [36]. Thus, regardless of the technical issues that have to be addressed (e.g. electromagnetic interference between the two systems, and MRI-based attenuation correction), PET/MRI may be a good alternative or complement to PET/CT for the evaluation of disease [37].

The technique is relatively easy to perform on compliant patients, and a full examination will take approximately 45-70 min, depending on the desired protocol and the size of the patient. PET and MRI data are acquired simultaneously, and both PET and multisequence MRI acquisitions are highly customizable. Whole-body scans are feasible, but bed positions vary depending on the patient's primary malignancy and the extent of the disease, and more localized examinations may also be performed, e.g. brain PET/MRI. Various diagnostic MRI sequences are acquired while simultaneously acquiring PET data. Imaging time is typically $6-8 \mathrm{~min}$ per bed position and is primarily dictated by the number of MRI pulse sequences that are to be acquired rather than the PET count statistics.

PET/MRI, like any new technology, has limitations. One of the most crucial steps will be the further improvement of MRI-based PET attenuation correction, which would allow for more accurate reconstructed images and more precise SUV calculations. Currently implemented attenuation correction sequences do not provide bone attenuation correction values. This limitation with regard to quantification originates from the lack of cortical bone signal on conventional MRI sequences and makes bone segmentation suboptimal in attenuation maps derived from MRI scans [38]. Ongoing research is seeking to resolve this issue by using ultra-short echo time (UTE) sequences, which allow the visualization of bones. Other drawbacks of MRI are its relative susceptibility to motion artefacts, as well as artefacts and signal loss, resulting from magnetic field inhomogeneities (particularly at air-tissue and air-bone interfaces). However, although MRI of certain organs such as the lungs and heart (coronary arteries) can be challenging, further technical developments and refinements in MRI sequence design may well resolve many of these issues in the foreseeable future. Nevertheless, in many body areas, including the central and peripheral nervous system, musculoskeletal system, head and neck, breast and parenchymal abdominal and pelvic organs (e.g. liver, uterus and prostate), MRI already has well established clinical advantages over CT. It is in these areas that the combination of PET/MRI is likely to have its first clinical 
Fig. 8 Combined (sequential) 7.0 T MRI and FDG PET performed in a 24-year-old male with unexplained medically refractory left frontocentral epilepsy showed a hyperintense (top left), non-gadoliniumenhanced (top right), calcified (bottom left), hypermetabolic (bottom right) lesion (arrows). The lesion was surgically removed and pathological examination revealed a $\mathrm{WHO}$ grade I ganglioglioma with leptomeningeal extension (color figure online)

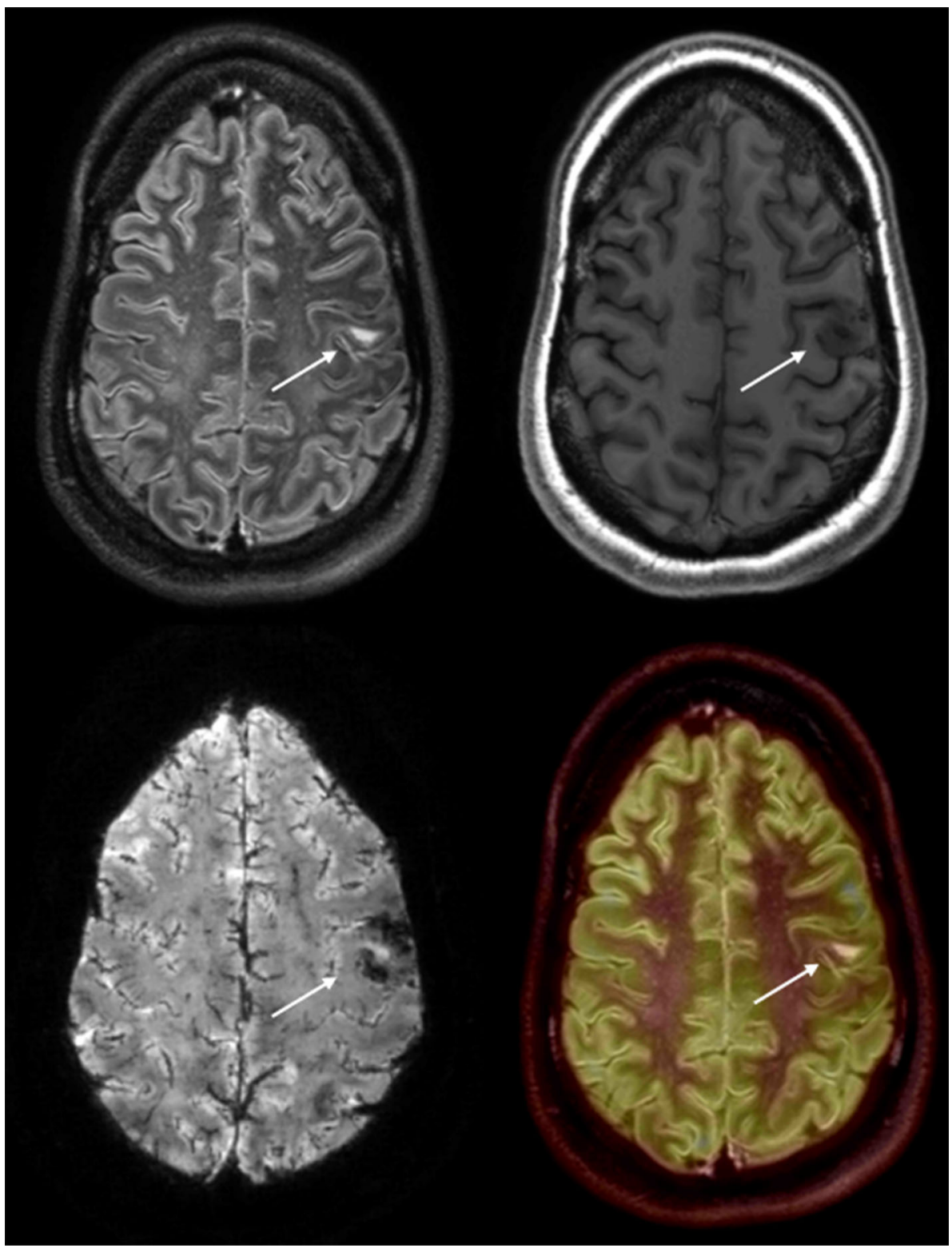

impact. Integrated PET/MRI technology is spreading rapidly, and several centres have published reports of their initial clinical experience with PET/MRI in different settings: oncology, paediatric oncology, intracranial masses and neuroimaging in general [39-43]. However, in order to establish evidence-based clinical indications for PET/MRI, it is imperative that better-designed studies in more homogeneous patient populations be performed.

It is hypothesized that PET/MRI will be a useful tool in the staging of lung cancer. The superior resolution and contrast of MRI allows for excellent delineation of the extent of the primary tumour (T-stage) and, compared with $\mathrm{CT}$, is a more accurate means of detecting chest wall invasion [44]. Furthermore, MRI is more accurate than CT in the detection of metastases in the brain and adrenals, both common sites of metastases from lung cancer. These advantages, combined with the excellent performance of PET in the detection of nodal metastases and the assessment of treatment response, suggest that PET/MRI has a promising future in lung cancer. Nevertheless, technical hurdles do remain, particularly with regard to the detection of subcentimetre pulmonary nodules. Advanced methods based on HASTE (half-Fourier-acquisition single-shot turbo spin-echo) and other MRI sequences currently under development may overcome this limitation.

Another area in which PET/MRI looks likely to establish a strong position is that of evaluation of the liver, as MRI has been shown to be a more sensitive modality for the detection of liver metastases. In a comparison of enhanced liver MRI and whole-body FDG PET for the 
detection of liver metastases, MRI and FDG PET showed sensitivities of 81.4 and $67.0 \%$ and accuracies of 75.5 and $64.1 \%$, respectively. Additionally, all subcentimetre lesions were identified on MRI, whereas less than half were detected on PET [45]. The authors postulate that PET/MRI will play a crucial role in the detection of liver metastases, the characterization of hepatic lesions and the assessment of treatment response in this organ.

There are many other potential oncological applications for PET/MRI, but they are beyond the scope of this brief review. A general concept to bear in mind is that the superior soft tissue contrast of MRI is likely to play a role in better delineating tumours in areas where CT is less accurate, such as in the pelvis and the brain. Advanced MRI sequences are expected to improve the staging and overall assessment of prostate cancer, rectal cancer and gynaecological malignancies. This setting will see exploitation of the high resolution of MRI for delineation of primary tumours and the high sensitivity of PET for detection of nodal and distant metastases. Improved contrast in the neck may possibly lead to improved diagnostic accuracy compared with CT.

PET/MRI is already starting to be employed in cardiovascular research. Protocols under development are expected to open the way for comprehensive assessment of cardiac function, coronary artery blood flow, cardiac inflammation, myocardial innervation and other physiological and pathophysiological processes in the heart. MRI will provide superior anatomical correlation and accurate information about perfusion and myocardial injury, and will also allow improved gating and quantification of PET tracers. The potential for future cardiac applications of simultaneous PET/MRI is vast.

Neuroimaging has always been an important field of application of both PET and MRI [46]. The first combined PET/MRI study in humans was a combined FDG PET/MRI brain study performed in two patients just after a PET/CT, performed for other clinical reasons [47]. No obvious interference between PET and MRI was observed, showing the feasibility of combined PET/MRI in the clinical setting. Extensive PET/MRI clinical research is under way for numerous neurological applications, including the evaluation of dementia, epilepsy and psychiatric disorders. Simultaneous data acquisition has the potential to offer a broad evaluation of cerebral pathophysiology on a molecular, anatomical and functional level [48]. More recent studies have confirmed that structural, functional and molecular imaging of patients with brain tumours is feasible using simultaneous PET/ MRI [49, 50], as the quality of MRI was uncompromised and a high concordance of PET results between PET/ MRI and PET/CT was observed (Fig. 8). Although no conclusions can be drawn from these studies, they encourage the application of this technique in further clinical studies in patients with brain tumours. It can be envisaged that PET/MRI will improve brain tumour

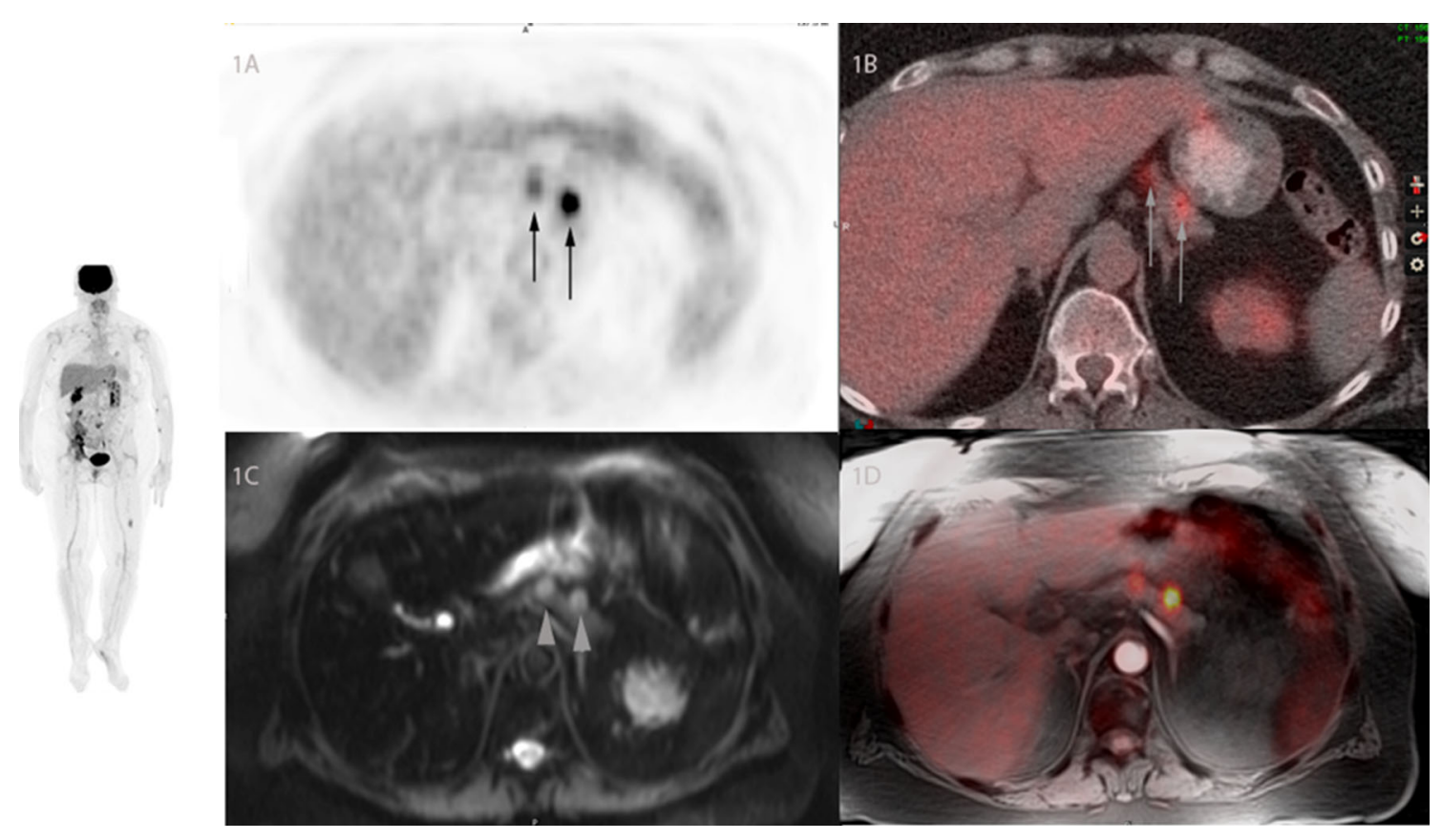

Fig. 9 53-Year-old male with sarcoma and two FDG-avid metastatic lesions (arrows) projecting to the body of the pancreas (a FDG PET. b fused FDG PET/CT), poorly seen on CT and clearly demarcated on DWI with restricted diffusion (c arrow head). Fused PET/MR demonstrates precise image co-registration (d fused T1 radial VIBE (volume interpolated breath-hold examination)/PET). This case illustrates the superior soft tissue contrast of PET/MRI for pancreatic lesions (color figure online) 
detection, delineation and grading, before, during and after therapy [45].

There are other settings in which PET/MRI may play an important role. These include the detection of seizure foci and the evaluation of neurodegenerative disorders. For example, it has already been reported that presurgical multimodality evaluation, including PET/MRI coregistration, improves non-invasive identification of subtle cortical dysplasia and subsequently allows successful surgical treatment [51]. Furthermore, in very mild AD, MRI as well as FDG PET showed a high diagnostic accuracy and hinted at the potential benefits of combining structural and functional images with hybrid PET/MRI [52].

It should also be noted that MRI data can be used for PVC of PET data. The clinical applications of correction techniques in neuroimaging are especially crucial in diseases involving atrophy, such as Alzheimer's disease [53]. Cho et al. [54] recently examined the power of combined ultra-high-resolution brain MRI and PET for exploring detailed structure and function of brain substructures important in human behaviour and memory. They measured glucose metabolism in hippocampal substructures by sequential FDG PET and 7.0 T MRI and demonstrated that measurement of glucose metabolism in individual thalamic nuclei is feasible. They also managed to visualize individual raphe nucleus groups within the brainstem and quantify their glucose metabolism; in the past, these were visible only through in vitro histological studies.
PET/MRI holds promise as a novel technology with applications in oncology, neurology and cardiology (Figs. 9, 10, 11). The use of MRI instead of CT allows improved lesion detection in selected applications, reduces radiation exposure, potentially improves quantification and opens up a vast array of possibilities for multiparametric assessment of disease and for individualization of patient care. Although current clinical experience is limited, preliminary results strongly suggest that hybrid PET/MRI will develop into a powerful tool for both whole-body and targeted examinations.

\section{Concluding remarks}

The global emergence and increasingly widespread implementation of hybrid FDG PET/CT, which combines anatomical and morphological data with functional and molecular data, has brought about a paradigm shift in the use of diagnostic imaging. Molecular imaging is now at the forefront of patient management on very many levels, not only in the initial diagnosis, but also in providing valuable tools for patient selection. It is used in surgery and other invasive procedures, as well as in monitoring of response to treatment, in many cases sparing patients futile therapeutic interventions that in addition to being costly can also subject patients to considerable risk. The application of the multiple-time-point imaging, PVC and novel quantitative
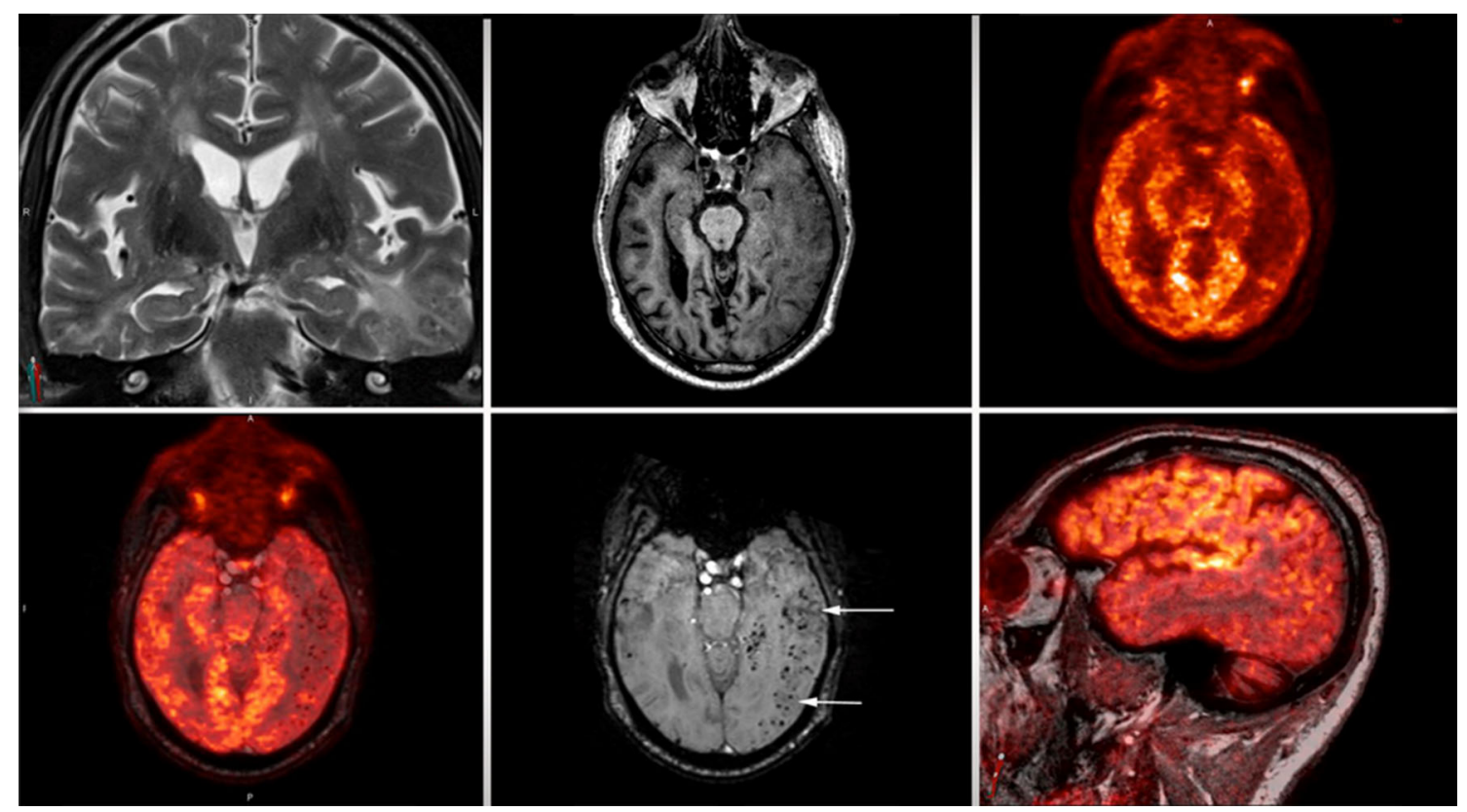

Fig. 10 71-year-old male with memory loss. Coronal T2 images (top left) demonstrate normal hippocampal volumes. Multisequence FDG PET/MRI demonstrates temporoparietal grey matter hypometabolism characteristic of $\mathrm{AD}$, left greater than right (top middle MRI, top right FDG PET, and bottom left fused FDG PET/MRI). SWI (susceptibility weighted imaging) sequence on MRI (bottom middle) and sagittal fused FDG PET/SWI (bottom right) demonstrates susceptibility artefacts (arrows - small hypointense foci) compatible with amyloid angiopathy superimposed on $\mathrm{AD}$ (color figure online) 

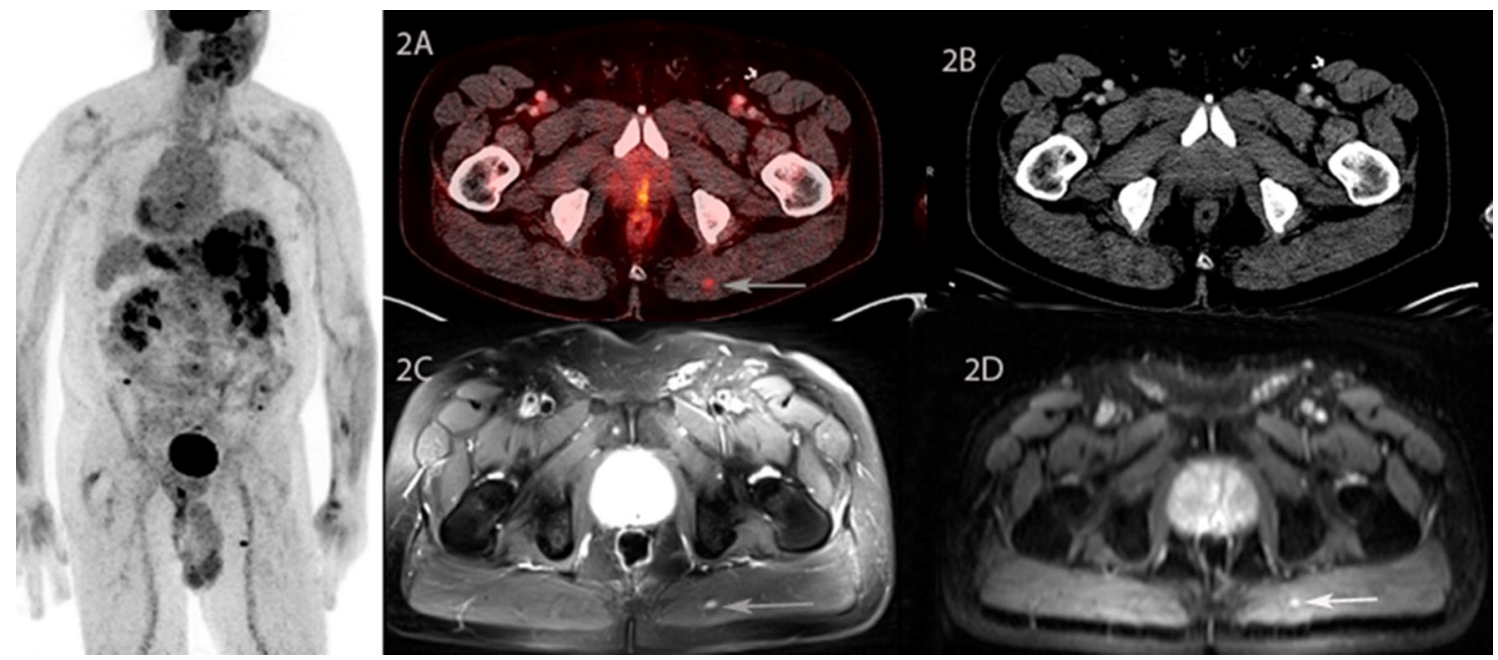

Fig. 11 73-Year-old male with melanoma presenting with focal FDG uptake in the left gluteus maximus (a fused PET/CT, arrow) without anatomical correlate on CT (b). FDG PET/MR shows T2 bright

methods described earlier will translate into enhanced sensitivity and specificity in variety of clinical settings, in which FDG PET imaging has not yet shown its full potential. In the coming years, we also expect to see PET/ MRI evolving as a powerful tool in neuroimaging, used in characterization of cancer in the liver, prostate, ovaries and adrenal glands, in studies of the musculoskeletal system and in the imaging of pregnant women, children and patients requiring multiple studies.

Conflict of interest The authors have no conflict of interest.

Ethical standard All authors contributed to conception and design of the article, drafting of the article, critical revision and final approval of the version to be published.

Human and animal rights This article does not contain any studies with human or animal subjects performed by any of the authors.

\section{References}

1. Mankoff DA (2007) A definition of molecular imaging. J Nucl Med 48(18N):21N

2. Hess S, Blomberg BA, Zhu H, Høilund-Carlsen PF, Alavi A (2014) The pivotal role of FDG-PET/CT in modern medicine. Acad Radiol 21:232-249

3. Portnow LH, Vaillancourt DE, Okun MS (2013) The history of cerebral PET scanning. From physiology to cutting-edge technology. Neurology 80:952-956

4. Høilund-Carlsen PF, Gerke O, Vilstrup MH et al (2011) PET/CT without capacity limitations: a Danish experience from a European perspective. Eur Radiol 21:1277-1285

5. Basu S, Kwee TC, Torigian D, Saboury B, Alavi A (2011) Suboptimal and inadequate quantification: an alarming crisis in medical application of PET. Eur $\mathrm{J}$ Nucl Med Mol Imaging 38:1381-1382

6. Basu S, Alavi A (2007) Partial volume correction of standardized uptake values and the dual time point in FDG-PET imaging: subcentimetre nodule (c arrow) with restricted diffusion on DWI (d), demonstrating the excellent soft tissue contrast and resolution of FDG PET/MR (color figure online)

should they be routinely employed in assessing patients with cancer? Eur J Nucl Med Mol Imaging 34:1527-1529

7. Basu S, Kwee TC, Surti S, Akin EA, Yoo D, Alavi A (2011) Fundamentals of PET and PET/CT imaging. Ann N Y Acad Sci 1228:1-18

8. Schillaci O (2012) Use of dual-point fluorodeoxyglucose imaging to enhance sensitivity and specificity. Semin Nucl Med 42:267-280

9. Basu S, Zaidi H, Holm S, Alavi A (2011) Quantitative techniques in PET-CT imaging. Curr Med Imaging Rev 7:216-233

10. Kwee TC, Torigian DA, Alavi A (2013) Overview of positron emission tomography, hybrid positron emission tomography instrumentation, and positron emission tomography quantification. J Thoracic Imaging 28:4-10

11. Minn H, Leskinen-Kallio S, Lindholm P et al (1993) $\left[{ }^{18} \mathrm{~F}\right]$ fluorodeoxyglucose uptake in tumours: kinetic vs. steady-state methods with reference to plasma insulin. J Comput Assist Tomogr 17:115-123

12. Kole A, Nieweg O, Pruim J et al (1997) Standardized uptake value and quantification of metabolism for breast cancer imaging with FDG and L- $\left[1-{ }^{11} \mathrm{C}\right]$ tyrosine PET. J Nucl Med 38:692-696

13. Zhuang HM, Cortés-Blanco A, Pourdehnad M et al (2001) Do high glucose levels have differential effect on FDG uptake in inflammatory and malignant disorders? Nucl Med Commun 22:1123-1128

14. Boellaard R, O'Doherty MJ, Weber WA et al (2010) FDG PET and PET/CT: EANM procedure guidelines for tumour PET imaging: version 1.0. Eur J Nucl Med Mol Imaging 37:181-200

15. Tomasi G, Turkheimer F, Aboagye E (2012) Importance of quantification for the analysis of PET data in oncology: review of current methods and trends for the future. Mol Imaging Biol 14:131-146

16. Sharif S, Zahid I, Routledge T, Scarci M (2011) Does positron emission tomography offer prognostic information in malignant pleural mesothelioma? Interact Cardiovasc Thorac Surg 12:806-811

17. Hongtao L, Hui Z, Bingshun W, Xiaojin W, Zhiyu W, Shuier Z, Aina H, Yuanjue S, Daliu M, Zan S, Yang Y (2011) ${ }^{18}$ F-FDG positron emission tomography for the assessment of histological response to neoadjuvant chemotherapy in osteosarcomas: a metaanalysis. Surg Oncol 21:e165-e170 
18. Nanni C, Boriani L, Salvadori C et al (2012) FDG PET/CT is useful for the interim evaluation of response to therapy in patients affected by haematogenous spondylodiscitis. Eur J Nucl Med Mol Imaging 39:1538-1544

19. Groshar D, Bernstine H, Stern D et al (2010) PET/CT enterography in Crohn disease: correlation of disease activity on CT enterography with ${ }^{18}$ F-FDG uptake. J Nucl Med 51:1009-1014

20. Rondina MT, Lam UT, Pendleton RC et al (2012) ${ }^{18}$ F-FDG PET in the evaluation of acuity of deep vein thrombosis. Clin Nucl Med 37:1139-1145

21. Hickeson M, Yun M, Matthies A et al (2002) Use of a corrected standardized uptake value based on lesion size on CT permits accurate characterization of lung nodules on FDG-PET. Eur $\mathbf{J}$ Nucl Med Mol Imaging 29:1639-1647

22. Avril N, Bense S, Ziegler SI et al (1997) Breast imaging with fluorine-18-FDG PET: quantitative image analysis. J Nucl Med 38:1186-1191

23. Lubberink M, Tolmachev V, Widstrom C, Bruskin A, Lundqvist $\mathrm{H}$, Westlin JE (2002) ${ }^{110} \mathrm{~m}$-In-DTPA-D-Phe ${ }^{1}$-octreotide for imaging of neuroendocrine tumors with PET. J Nucl Med 43:1391-1397

24. Cheng G, Alavi A, Lim E et al (2013) Dynamic changes of FDG uptake and clearance in normal tissues. Mol Imaging Biol $15: 345-352$

25. Zhuang H, Pourdehnad M, Lambright ES et al (2001) Dual time point ${ }^{18} \mathrm{~F}$-FDG PET imaging for differentiating malignant from inflammatory processes. J Nucl Med 42:1412-1417

26. Basu S, Kung J, Houseni M, Zhuang H, Tidmarsh GF, Alavi A (2009) Temporal profile of fluorodeoxyglucose uptake in malignant lesions and normal organs over extended time periods in patients with lung carcinoma: implications for its utilization in assessing malignant lesions. Q J Nucl Med Mol Imaging 53:9-16

27. Matthies A, Hickeson M, Cuchiara A, Alavi A (2002) Dual time point ${ }^{18} \mathrm{~F}$-FDG PET for the evaluation of pulmonary nodules. J Nucl Med 43:871-875

28. Kumar R, Loving VA, Chauhan A, Zhuang H, Mitchell S, Alavi A (2005) Potential of dual-time-point imaging to improve breast cancer diagnosis with ${ }^{18} \mathrm{~F}$-FDG PET. J Nucl Med 46:1819-1824

29. Hustinx R, Smith RJ, Benard F et al (1999) Dual time point fluorine-18 fluorodeoxyglucose positron emission tomography: a potential method to differentiate malignancy from inflammation and normal tissue in the head and neck. Eur $\mathrm{J}$ Nucl Med 26:1345-1348

30. Lee JW, Kim SK, Lee SM, Moon SH, Kim TS (2011) Detection of hepatic metastases using dual-time-point FDG PET/CT scans in patients with colorectal cancer. Mol Imaging Biol 13:565-572

31. Alavi A, Reivich M, Greenberg J et al (1981) Mapping of functional activity in brain with ${ }^{18} \mathrm{~F}$-fluoro-deoxyglucose. Semin Nucl Med 11:24-31

32. Reivich M, Alavi A, Wolf A et al (1982) Use of 2-deoxy$\mathrm{D}\left[1-{ }^{11} \mathrm{C}\right]$ glucose for the determination of local cerebral glucose metabolism in humans: variation within and between subjects. J Cereb Blood Flow Metab 2:307-319

33. Basu S, Saboury B, Torigian DA, Alavi A (2011) Current evidence base of FDG-PET/CT imaging in the clinical management of malignant pleural mesothelioma: emerging significance of image segmentation and global disease assessment. Mol Imaging Biol 13:801-811

34. Basu S, Saboury B, Werner T, Alavi A (2011) Clinical utility of FDG-PET and PET/CT in non-malignant thoracic disorders. Mol Imaging Biol 13:1051-1060

35. Van de Wiele C, Kruse V, Smeets P, Sathekge M, Maes A (2013) Predictive and prognostic value of metabolic tumour volume and total glycolysis in solid tumours. Eur J Nucl Med Mol Imaging 40:290-301

36. Kwee TC, Takahara T, Klomp DW, Luijten PR (2010) Cancer imaging: novel concepts in clinical magnetic resonance imaging. J Intern Med 268:120-132

37. Torigian DA, Zaidi H, Kwee TC et al (2013) PET/MRI: technical aspects and potential clinical applications. Radiology 267:26-44

38. Samarin A, Burger C, Wollenweber SD et al (2012) PET/MR imaging of bone lesions-implications for PET quantification from imperfect attenuation correction. Eur J Nucl Med Mol Imaging 39:1154-1160

39. Boss A, Bisdas S, Kolb A et al (2010) Hybrid PET/MRI of intracranial masses: initial experiences and comparison to PET/ CT. J Nucl Med 51:1198-1205

40. Drzezga A, Souvatzoglou M, Eiber M et al (2012) First clinical experience with integrated whole-body PET/MR: comparison to PET/CT in patients with oncologic diagnoses. J Nucl Med 53:845-855

41. Garibotto V, Heinzer S, Vulliemoz S et al (2013) Clinical applications of hybrid PET/MRI in neuroimaging. Clin Nucl Med 38:e13-e18

42. Hirsch FW, Sattler B, Sorge I et al (2013) PET/MR in children. Initial clinical experience in paediatric oncology using an integrated PET/MR scanner. Pediatr Radiol 43:860-875

43. Kjær A, Loft A, Law I et al (2013) PET/MRI in cancer patients: first experiences and vision from Copenhagen. MAGMA 36:37-47

44. Akata S, Kajiwara N, Park J et al (2008) Evaluation of chest wall invasion by lung cancer using respiratory dynamic MR. J Med Imaging Radiat Oncol 52:36-39

45. Sahani D, Kalva S, Fischman A et al (2005) Detection of liver metastases from adenocarcinoma of the colon and pancreas: comparison of mangafodipir trisodium-enhanced liver MR and whole-body FDG-PET. AJR Am J Roentgenol 185(1):239-246

46. Kwee TC, Donswijk ML (2013) Application of advanced MR imaging techniques and the evolving role of PET/MR imaging in neuro-oncology. PET Clin 8:183-199

47. Schlemmer HP, Pichler BJ, Schmand M et al (2008) Simultaneous MR/PET imaging of the human brain: feasibility study. Radiology 248:1028-1035

48. Stegger L, Martirosian P, Schwenzer N et al (2012) Simultaneous PET/MR imaging of the brain: feasibility of cerebral blood flow measurements with FAIR-TrueFISP arterial spin labeling MRI. Acta Radiol 53:1066-1072

49. Boss A, Bisdas S, Kolb A et al (2010) Hybrid PET/MRI of intracranial masses: initial experiences and comparison to PET/ CT. J Nucl Med 51:1198-1205

50. Schwenzer NF, Stegger L, Bisdas S et al (2012) Simultaneous PET/ MR imaging in a human brain PET/MR system in 50 patientscurrent state of image quality. Eur J Radiol 81:3472-3478

51. Salamon N, Kung J, Shaw SJ et al (2008) FDG-PET/MRI coregistration improves detection of cortical dysplasia in patients with epilepsy. Neurology 71:1594-1601

52. Kawachi T, Ishii K, Sakamoto S et al (2006) Comparison of the diagnostic performance of FDG-PET and VBM-MRI in very mild Alzheimer's disease. Eur J Nucl Med Mol Imaging 33:801-809

53. Erlandsson K, Buvat I, Pretorius PH, Thomas BA, Hutton BF (2012) A review of partial volume correction techniques for emission tomography and their applications in neurology, cardiology and oncology. Phys Med Biol 57:R119-R159

54. Cho ZH, Son YD, Choi EJ et al (2013) In-vivo human brain molecular imaging with a brain-dedicated PET/MRI system. MAGMA 26:71-79 